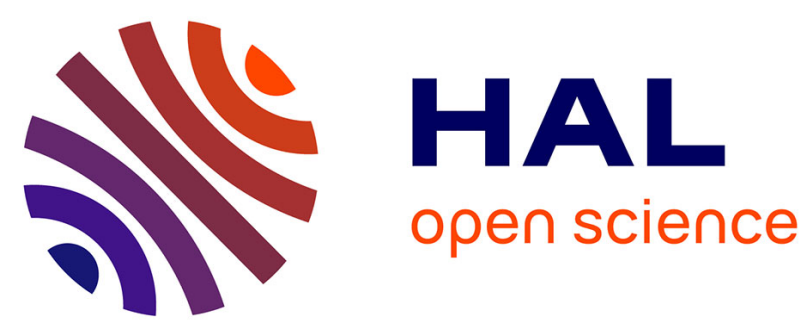

\title{
Measurement techniques of the sensitivity functions to characterize the vibration response of panels under turbulent boundary layer excitation
}

\author{
Christophe Marchetto, Laurent Maxit, Olivier Robin, Alain Berry
}

\section{To cite this version:}

Christophe Marchetto, Laurent Maxit, Olivier Robin, Alain Berry. Measurement techniques of the sensitivity functions to characterize the vibration response of panels under turbulent boundary layer excitation. Flinovia-Flow Induced Noise and Vibration Issues and Aspects-II: A Focus on Measurement, Modeling, Simulation and Reproduction of the Flow Excitation and Flow Induced Response, 2018. hal-02121084

\author{
HAL Id: hal-02121084 \\ https://hal.science/hal-02121084
}

Submitted on 6 May 2019

HAL is a multi-disciplinary open access archive for the deposit and dissemination of scientific research documents, whether they are published or not. The documents may come from teaching and research institutions in France or abroad, or from public or private research centers.
L'archive ouverte pluridisciplinaire HAL, est destinée au dépôt et à la diffusion de documents scientifiques de niveau recherche, publiés ou non, émanant des établissements d'enseignement et de recherche français ou étrangers, des laboratoires publics ou privés. 


\title{
Measurement techniques of the sensitivity functions to characterize the vibration response of panels under turbulent boundary layer excitation
}

\author{
Christophe Marchetto ${ }^{1,2}$, Laurent Maxit ${ }^{1}$, Olivier Robin ${ }^{2}$, and Alain Berry ${ }^{2}$ \\ 1 Univ Lyon, INSA-Lyon, Laboratoire Vibrations Acoustique, F-69621 Villeurbanne, \\ France, \\ christophe.marchetto@usherbrooke.ca, \\ laurent.maxit@insa-lyon.fr, \\ 2 Groupe d'Acoustique de l'Université de Sherbrooke, Université de Sherbrooke, \\ Sherbrooke, J1K 2R1, Canada, \\ olivier.robin@usherbrooke.ca, \\ alain.berry@usherbrooke.ca
}

\begin{abstract}
This study aims at developing an experimental method for characterizing the vibroacoustic behavior of panels excited by random pressure fields. Although the method would be theoretically applicable to any stationary in time and spatially homogeneous random process, and for points belonging to the acoustic medium or to the panel, the turbulent boundary layer excitation is considered in this study while considering the vibration response exclusively. The interest of industrials towards this excitation has grown over the years. The main reasons being that the associated test means (i.e. wind tunnel or in situ measurements) are hard to control and very expensive. They are also subjected to large variabilities between laboratories, which makes it hard to attest the validity of the measuring technique. The proposed method allows to experimentally characterize a panel under such an excitation by separating the contribution of the excitation from the vibration behavior of the panel.
\end{abstract}

Keywords: panel vibration, turbulent boundary layer, sensitivity functions, reciprocity principles

\section{Introduction}

The experimental vibration characterization of panels under a turbulent boundary layer (TBL) excitation is of great interest for the transport industry. The usual associated test means (wind tunnel or in situ measurements) are hard to control, very costly and subjected to variabilities between laboratories and/or measuring techniques. A considerable work has been conducted to experimentally characterize the vibration behavior of panels under TBL excitations without the use of those usual test means. Most of the recently developed methods consist 
in synthesizing the pressure field induced by a TBL using an array of acoustic sources [1-6]. The common issues of those methods are: the number of sources required to reproduce small correlation lengths, which becomes prohibitive at high frequency, and/or the inability to accurately reproduce the induced pressure field outside the acoustic wavenumber domain, which is the predominant domain for subsonic TBL.

In this context, the aim of this study is to investigate an alternative and robust approach to experimentally characterize the response of panels to a TBL excitation by separating the contributions of the forcing excitation and those of the dynamic behavior of the panel. Indeed, the mathematical formulation of a panel vibration response when submitted to random excitations in the wavenumber domain allows estimating the system response at any point on the structure from wall-pressure cross spectral density (CSD) functions (characterizing the excitation) and from so-called 'sensitivity functions'. The latter are defined as the panel response to wall-pressure acoustic plane waves and characterize the panel vibration behavior. The estimation of the panel response to a TBL excitation therefore only requires the experimental measurement of sensitivity functions in the acoustic wavenumber domain and an estimation of the wall-pressure CSD functions of the considered excitation.

A method is proposed for estimating the sensitivity functions experimentally. Whereas the direct interpretation of the sensitivity functions would require exciting the panel by sets of wall plane waves, which is not easy from an experimental point of view, an alternative method based on the reciprocity principle is proposed. The reciprocity principle states that the sensitivity functions at any point on the structure are equivalent to the panel velocity response expressed in the wavenumber domain when the system is excited by a normal force at the same point. The proposed experimental process consists in exciting the panel with a normal force at the point where the panel response is to be determined. The spatial vibratory response of the panel is then measured with a scanning laser vibrometer. In a subsequent post-processing phase, a discrete 2 -D wavenumber transform of the measured vibratory field is performed to deduce the sensitivity functions. Finally, using measured wall-pressure fluctuations and the previously estimated sensitivity functions, the response of the panel when excited by a TBL can be deduced at the point of interest.

The paper is organized as follows: the mathematical formulation of the vibroacoustic problem is presented in Sec. 2.1 where the sensitivity functions involved in the problem are defined. An alternative interpretation of these functions based on the reciprocity principle is proposed in Sec. 2.2. This interpretation suggests a simple implementation for measuring the sensitivity functions. The proposed methodology for characterizing the panel response under a turbulent boundary layer is summarized in Sec. 3. An experimental validation of the determination of the sensitivity functions is provided in Sec. 4. Finally, a comparison with measurements performed in an anechoic wind tunnel is shown in Sec. 5 along with an experimental characterization of the wall-pressure fluctuations induced by the reproduced TBL. 


\section{Vibration behavior of panels under turbulent boundary layer}

Let us consider a baffled panel of surface $\Sigma_{p}$ with arbitrary boundary conditions separating two semi-infinite acoustic domains. As shown in Fig. 1, one supposes that a fully developed TBL is exciting the panel on one side. The TBL is characterized by its flow velocity $U_{\infty}$ outside the boundary layer and is considered stationary in time and spatially homogeneous. We also define two points on the panel surface $(z=0), \mathbf{x}=(x, y)$ the observation point and $\tilde{\mathbf{x}}=(\tilde{x}, \tilde{y})$ the excitation point. Both points are defined in the Cartesian coordinate system $(x, y, z)$ with the origin at the center of the panel shown in Fig. 1.

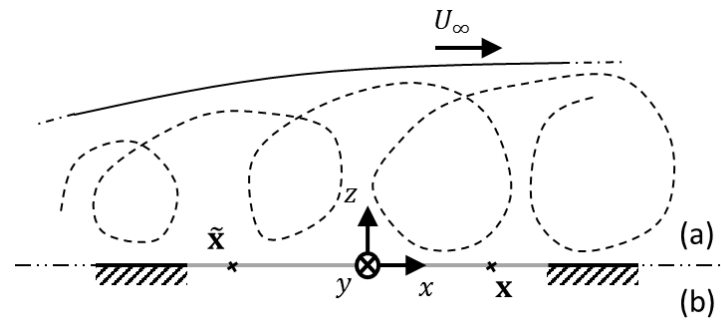

Fig. 1. Panel (gray line) and coordinate system. (a) source side: TBL excitation. (b) receiving side: semi-infinite domain.

To experimentally characterize the vibration response of this panel, the onesided normal velocity frequency response $v$ at a given point on the panel is considered. As the excitation is random, the velocity response is derived from the auto spectral density (ASD) function of the normal velocity $G_{v v}(\mathbf{x}, f)$. An approach for evaluating this quantity based on deterministic transfer functions and using a reciprocity principle is presented in Secs. 2.1 and 2.2.

\subsection{Mathematical formulation of the vibration response}

Let us consider the blocked wall-pressure field $p_{b}(\tilde{\mathbf{x}}, t)$ exerted on the panel by a TBL excitation at point $\tilde{\mathbf{x}}$ as a function of time. The vibration response of the panel at point $\mathbf{x}$ when the panel is excited by $p_{b}(\tilde{\mathbf{x}}, t)$ is denoted $v(\mathbf{x}, t)$. This response can be expressed by the convolution product [7]

$$
v(\mathbf{x}, t)=\iint_{\Sigma_{p}} \int_{-\infty}^{\infty} h_{v / F_{n}}(\mathbf{x}, \tilde{\mathbf{x}}, t-\tau) p_{b}(\tilde{\mathbf{x}}, \tau) d \tau d \tilde{\mathbf{x}},
$$

where $h_{v / F_{n}}(\mathbf{x}, \tilde{\mathbf{x}}, t)$ is the structural velocity impulse response at point $\mathbf{x}$ for a normal unit force applied at point $\tilde{\mathbf{x}}$. Assuming that the random process is 
ergodic, the cross-correlation function $R_{v v}(\mathbf{x}, t)$ is defined by

$$
R_{v v}(\mathbf{x}, t)=\int_{-\infty}^{\infty} v(\mathbf{x}, \tau) v(\mathbf{x}, t+\tau) d \tau .
$$

Introducing Eq. (1) in Eq. (2) and performing a time Fourier transform of the resulting expression of the cross-correlation function gives the space-frequency spectrum $G_{v v}(\mathbf{x}, f)$, which after some manipulations (see [7] for details) can be written as

$$
G_{v v}(\mathbf{x}, f)=\iint_{\Sigma_{p}} \iint_{\Sigma_{p}} H_{v / F_{n}}(\mathbf{x}, \tilde{\mathbf{x}}, f) H_{v / F_{n}}^{*}(\mathbf{x}, \tilde{\tilde{\mathbf{x}}}, f) G_{p_{b} p_{b}}(\tilde{\mathbf{x}}, \tilde{\tilde{\mathbf{x}}}, f) d \tilde{\mathbf{x}} d \tilde{\tilde{\mathbf{x}}}
$$

where $H_{v / F_{n}}(\mathbf{x}, \tilde{\mathbf{x}}, f)$ is the time Fourier transform of $h_{v / F_{n}}(\mathbf{x}, \tilde{\mathbf{x}}, t)$ and corresponds to the panel velocity frequency response function at point $\mathbf{x}$ when it is excited by a normal force $F_{n}$ applied at point $\tilde{\mathbf{x}}$, and $G_{p_{b} p_{b}}(\tilde{\mathbf{x}}, \tilde{\tilde{\mathbf{x}}}, f)$ is the time Fourier transform of the cross-correlation function of the blocked wall-pressure. Defining the wavenumber-frequency spectrum of the wall-pressure $G_{p_{b} p_{b}}(\boldsymbol{k}, f)$ as the wavenumber transform of the space-frequency spectrum $G_{p_{b} p_{b}}(\tilde{\mathbf{x}}, \tilde{\tilde{\mathbf{x}}}, f)$,

$$
G_{p_{b} p_{b}}(\tilde{\mathbf{x}}, \tilde{\tilde{\mathbf{x}}}, f)=\frac{1}{4 \pi^{2}} \iint_{-\infty}^{\infty} G_{p_{b} p_{b}}(\boldsymbol{k}, f) e^{j \boldsymbol{k}(\tilde{\tilde{\mathbf{x}}}-\tilde{\mathbf{x}})} d \boldsymbol{k} .
$$

where $\boldsymbol{k}=\left(k_{x}, k_{y}\right)$ is the wavevector defined in the plane $(x, y)$ and $d \boldsymbol{k}$ is the two-dimensional wavenumber element. By introducing Eq. (4) in Eq. (3) and rearranging the terms, we get

$$
G_{v v}(\mathbf{x}, f)=\frac{1}{4 \pi^{2}} \iint_{-\infty}^{\infty}\left|H_{v}(\mathbf{x}, \boldsymbol{k}, f)\right|^{2} G_{p_{b} p_{b}}(\boldsymbol{k}, f) d \boldsymbol{k} .
$$

In practice, this integral is approximated considering a set of wavevectors in an appropriately defined wavenumber domain $\Omega_{\boldsymbol{k}}$ and using the rectangular integration rule. Eq. (5) shows that the panel has a filtering effect on the excitation [7], which somehow limits the wavenumber domain $\Omega_{\boldsymbol{k}}$ while ensuring a correct estimation of the integral (see Sec. 4.1). The one-sided frequency ASD function of the velocity at point $\mathbf{x}$ is thereby estimated with

$$
G_{v v}(\mathbf{x}, f) \approx \frac{1}{4 \pi^{2}} \sum_{\boldsymbol{k} \in \Omega_{\boldsymbol{k}}}\left|H_{v}(\mathbf{x}, \boldsymbol{k}, f)\right|^{2} G_{p_{b} p_{b}}(\boldsymbol{k}, f) \delta \boldsymbol{k},
$$

where $\delta \boldsymbol{k}$ represents the wavenumber resolution and $G_{p_{b} p_{b}}(\boldsymbol{k}, f)$ is the one-sided frequency CSD function of the blocked wall-pressure.

The $H_{v}(\mathbf{x}, \boldsymbol{k}, f)$ functions are called the sensitivity functions [8] and characterize the vibration behavior of the panel. They are defined by

$$
H_{v}(\mathbf{x}, \boldsymbol{k}, f)=\iint_{\Sigma_{p}} H_{v / F_{n}}(\mathbf{x}, \tilde{\mathbf{x}}, f) e^{-j \boldsymbol{k} \tilde{\mathbf{x}}} d \tilde{\mathbf{x}}
$$


where $H_{v / F_{n}}(\mathbf{x}, \tilde{\mathbf{x}}, f)$ is the time Fourier transform of $h_{v / F_{n}}(\mathbf{x}, \tilde{\mathbf{x}}, t)$ and, therefore, corresponds to the transfer function between the panel velocity and the applied force in the frequency domain. The sensitivity functions can be interpreted directly from Eq. (7) as the velocity frequency response at point $\mathbf{x}$ when the panel is excited by a wall-pressure plane wave of wavevector $\boldsymbol{- k}$ (i.e., due to the pressure field $\left.e^{-j \boldsymbol{k} \tilde{\mathbf{x}}}\right)$. This direct interpretation is depicted in Fig. 2(a).

The sensitivity functions must, therefore, be estimated only at the point of interest $\mathrm{x}$ and for the set of wavevectors in $\Omega_{\boldsymbol{k}}$. From an experimental point of view, wall-pressure plane waves cannot be easily reproduced, especially at high wavenumbers. To circumvent these issues, another interpretation of these sensitivity functions based on the reciprocity principle is given in Sec. 2.2.

\subsection{Sensitivity functions based on the reciprocity principle}

In order to propose another interpretation of the sensitivity functions, let us consider the standard reciprocity principle which states that the response of a system is invariant with respect to the exchange of points of excitation and observed response [9]. For the particular case of a normal force applied at point $\tilde{\mathbf{x}}$ and normal velocity observed at point $\mathbf{x}$, the reciprocity relationship can be translated following the previous notation into

$$
H_{v / F_{n}}(\mathbf{x}, \tilde{\mathbf{x}}, f)=H_{v / F_{n}}(\tilde{\mathbf{x}}, \mathbf{x}, f),
$$

Introducing Eq. (8) in Eq. (7) one obtains

$$
H_{v}(\mathbf{x}, \boldsymbol{k}, f)=\iint_{\Sigma_{p}} H_{v / F_{n}}(\tilde{\mathbf{x}}, \mathbf{x}, f) e^{-j \boldsymbol{k} \tilde{\mathbf{x}}} d \tilde{\mathbf{x}}
$$

The right hand side of Eq. (9) can be interpreted as the space-wavenumber transform of $H_{v / F_{n}}(\tilde{\mathbf{x}}, \mathbf{x}, f)$ with respect to the spatial variable $\tilde{\mathbf{x}}$. The points $\tilde{\mathbf{x}}$ become observation points on the panel surface $\Sigma_{p}$, which means that the space-wavenumber transform is performed over the vibration velocity field of the panel.

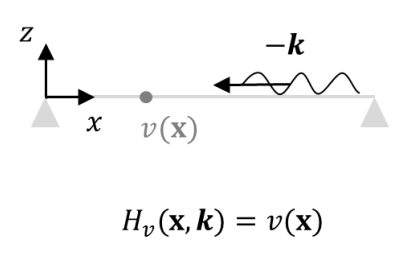

(a)

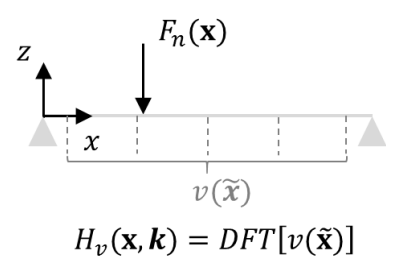

(b)

Fig. 2. Sensitivity functions $H_{v}$ : (a) direct interpretation and (b) reciprocal interpretation. 
To sum up, the sensitivity function $H_{v}(\mathbf{x}, \boldsymbol{k}, f)$ may be obtained by exciting the panel with a normal effort $F_{n}$ of unit amplitude at point $\mathbf{x}$ and by calculating the space-wavenumber transform of the panel velocity frequency response (as illustrated in Fig. 2(b)).

In practice, the vibratory field has to be measured on a regular grid of points denoted $\Gamma_{\tilde{\mathbf{x}}}$, using a scanning laser vibrometer, for example. The spacewavenumber transform is therefore approximated by a discrete Fourier transform (DFT). In order to avoid aliasing effects, the spatial resolution $\delta \tilde{\mathbf{x}}$ over $\Gamma_{\tilde{\mathbf{x}}}$ should be determined so that the spatial variations of the vibratory field can be correctly represented by the grid of points. For a homogeneous isotropic thin panel, $\delta \tilde{\mathbf{x}}$ should be less than or equal to a quarter of the natural flexural wavelength of the panel $\lambda_{f}$ at the highest frequency of interest. For a more complex panel, a preliminary study should be carried out to define this parameter (for instance, by using a numerical model of the panel or by using a trial and error procedure).

\section{Description of the proposed methodology}

A methodology for experimentally estimating the vibration response of a panel excited by a TBL is now presented. This methodology for evaluating the velocity ASD function $G_{v v}$ at a given point $\mathbf{x}$ of the panel $(z=0)$ is based on Eq. (6) and the second interpretation of the sensitivity functions, as described in Sec. 2.2. It can be summarized as follows:

- Excite the panel with a normal mechanical force at point $\mathbf{x}$ (for instance by using a shaker) and measure the normal velocity response of the panel at points $\tilde{\mathbf{x}} \in \Gamma_{\tilde{\mathbf{x}}}$ to determine $H_{v / F_{n}}(\tilde{\mathbf{x}}, \mathbf{x}, f)$,

- Perform a DFT of the panel velocity response $H_{v / F_{n}}(\tilde{\mathbf{x}}, \mathbf{x}, f)$ (with respect to $\tilde{\mathbf{x}})$ to obtain the sensitivity functions $H_{v}(\mathbf{x}, \boldsymbol{k}, f)$ at point $\mathbf{x}$ for $\boldsymbol{k} \in \Omega_{\boldsymbol{k}}$,

- Use Eq. (6) and a model or measurement of the wall-pressure CSD function of the TBL excitation to estimate the velocity ASD function $G_{v v}$ at point $\mathbf{x}$ (in the present paper, measured wall-pressure CSD functions are used to estimate the vibration response, they are presented in Sec. 5.1).

\section{Experimental validation of the proposed approach}

\subsection{Test case description}

For numerical and experimental validation purposes a test case is considered, which consists in a rectangular thin aluminum plate, simply supported on its four edges and submitted to a TBL excitation on one side. The plate geometrical and mechanical properties are detailed in Table 1. A mean value of the structural loss factor $\eta=0.005$ has been measured using the $-3 \mathrm{~dB}$ bandwidth method and taken into account in the numerical simulations. Simply-supported boundary conditions have been chosen because they lead to a simple analytical solution of the plate equation of motion. In addition, the experimental setup proposed 


\begin{tabular}{lc} 
Parameter (Symbol), Unit & Value \\
\hline Young's modulus $(E), \mathrm{GPa}$ & 68.9 \\
Poisson's ratio $(\nu)$ & 0.3 \\
Mass density $(\rho), \mathrm{kg} / \mathrm{m}^{3}$ & 2740 \\
Length $\left(L_{x}\right), \mathrm{mm}$ & 480 \\
Width $\left(L_{y}\right), \mathrm{mm}$ & 420 \\
Thickness $(h), \mathrm{mm}$ & 3.17
\end{tabular}

Table 1. Properties of the simply supported aluminum plate.

by Robin et al. [10] for reproducing these boundary conditions has already been validated.

The considered excitation is a TBL-like excitation reproduced in a low-speed anechoic wind tunnel (Mach $\leq 0.12$ ) at a flow velocity $U_{\infty}=40 \mathrm{~m} . \mathrm{s}^{-1}$. The frequency range of interest is $[170,2000 \mathrm{~Hz}]$ with a frequency resolution of 0.625 $\mathrm{Hz}$. This frequency range is well above the aerodynamic coincidence frequency $f_{c}$, given by

$$
f_{c}=\frac{U_{c}^{2}}{2 \pi} \sqrt{\frac{\rho h}{D}},
$$

where $D=\frac{E h^{3}}{12\left(1-\nu^{2}\right)}$ is the flexural stiffness and $U_{c}=0.75 \times U_{\infty}$ is the convection speed, assumed constant with the frequency. For the considered case, $f_{c} \approx 30 \mathrm{~Hz}$. It has been shown, through Eq. (5), that the plate filters out the TBL excitation above $f_{c}$. To explain this effect, the theoretical sensitivity functions have been plotted in Fig. 3(a) at point $\mathbf{x}_{M}$, for wavenumbers along $k_{x}\left(k_{y}=0\right)$ and as a function of the frequency. The strongly decreasing magnitude of $H_{v}\left(\mathbf{x}_{M}, k_{x}, f\right)$ above the flexural wavenumber can be noticed. Similarly, the Corcos model for $G_{p_{b} p_{b}}$ in the wavenumber domain [11] (which seems to correctly represent the wall-pressure fuctuations measured in the wind tunnel, see Sec. 5.1) is plotted in Fig. 3(b). Strong contributions around the convective wavenumber $k_{c}=\omega / U_{c}$ can be noticed. Fig. 3(c) shows the product of the squared sensitivity functions and the wall-pressure CSD functions (which is involved in the calculation of the vibration response of the plate), normalized by the maximum value at each frequency. For this particular case, the excitation is not completely filtered out as significant contributions from the TBL (along $k_{c}$ ) can be noticed in Fig. 3(c). It should be noted that in this paper the excitation is a low-speed subsonic TBL. The higher the flow velocity is, the lower the slope of the curve $k_{c}(f)$ and therefore, the less the plate filtering effect is effective. It can be directly related to the fact that, for a given plate with fixed parameters, $f_{c}$ increases with the flow velocity.

For the considered test case and reproduced TBL, a preliminary study (not shown here) indicated that the convective peak was almost entirely filtered out on the whole considered frequency range, which means that the vibration response to the reproduced TBL is mainly driven by the region inside the circle of radius $k_{f}$. Although defining the limit of the wavenumber domain by the flexural 
wavenumber (at $2000 \mathrm{~Hz}, k_{f} \approx 51 \mathrm{~m}^{-1}$ ) would probably lead to satisfying results, we have voluntarily retained the worst case scenario and fixed the wavenumber domain $\Omega_{\boldsymbol{k}}$ over which the sensitivity functions should be determined as follows:

$$
\begin{aligned}
k_{x}^{+} \simeq 113 \mathrm{~m}^{-1} ; k_{x}^{-} & =\simeq-66 \mathrm{~m}^{-1} \\
k_{y}^{+} \simeq 66 \mathrm{~m}^{-1} ; k_{y}^{-} & =\simeq-66 \mathrm{~m}^{-1}
\end{aligned}
$$

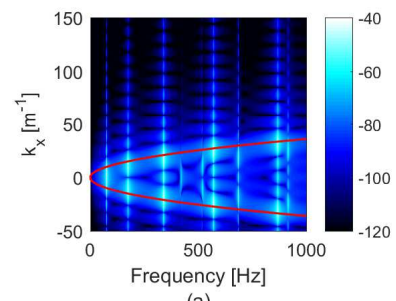

(a)

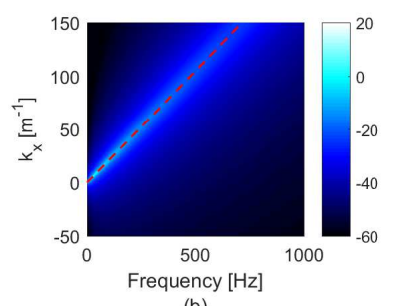

(b)

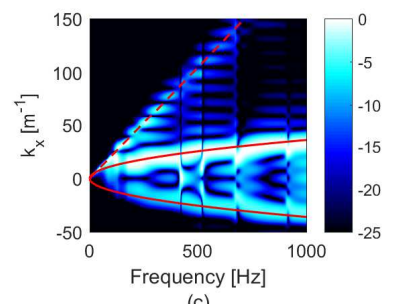

(c)

Fig. 3. Illustration of the filtering effect of the plate. (a) Theoretical sensitivity functions $H_{v}\left(\mathbf{x}_{M}, k_{x}, 0, f\right)(\mathrm{dB})$. (b) Corcos model $G_{p_{b} p_{b}}\left(k_{x}, 0, f\right)(\mathrm{dB}), U_{\infty}=40 \mathrm{~m} \cdot \mathrm{s}^{-1}$. (c) Product $\left|H_{v}\left(\mathbf{x}_{M}, k_{x}, 0, f\right)\right|^{2} \times G_{p_{b} p_{b}}\left(k_{x}, 0, f\right)(\mathrm{dB})$ normalized by the maximum at each frequency. Continuous red line: $k_{f}$. Dashed red line: $k_{c}$.

\subsection{Determination of the sensitivity functions}

In this section, we focus on the sensitivity functions $H_{v}$ at point $\mathbf{x}_{M}$ of coordinates $(x=0.18 \mathrm{~m}, y=0.09 \mathrm{~m})$ on the plate. To apply the methodology described in Sec. 3, the panel velocity field has to be measured or calculated on a grid of points $\Gamma_{\tilde{\mathbf{x}}}$. In the following, a uniform mesh of $37 \times 27$ points is considered in directions $x$ and $y$ respectively and a gap of $10 \mathrm{~mm}$ along the edges is left for practical reasons. This leads to a spatial separation of $\delta_{x} \approx 12.8 \mathrm{~mm}$ and $\delta_{y}=15.4 \mathrm{~mm}$ and ensures at least 4 points per flexural wavelength for all frequencies of interest. The highest wavenumbers $k_{x}^{\max }$ and $k_{y}^{\max }$ that can be resolved in directions $x$ and $y$, respectively, are given by

$$
k_{x}^{\max }=\frac{\pi}{\delta_{x}} \simeq 246 \mathrm{~m}^{-1} ; k_{y}^{\max }=\frac{\pi}{\delta_{y}} \simeq 204 \mathrm{~m}^{-1}
$$

These wavenumbers are well above twice the maximum wavenumbers $k_{x}^{+}$ and $k_{y}^{+}$. As a consequence, the considered grid of points provides correct estimation of the sensitivity functions in the considered wavenumber domain $\Omega_{\boldsymbol{k}}$. The wavenumber resolutions $\delta k_{x}$ and $\delta k_{y}$ in directions $x$ and $y$ respectively, are given by 


$$
\delta k_{x}=\frac{2 \pi}{L_{x}} \simeq 13 \mathrm{~m}^{-1} ; \delta k_{y}=\frac{2 \pi}{L_{y}} \simeq 15 \mathrm{~m}^{-1} .
$$

These wavenumber resolutions are relatively large because of the small dimensions of the panel. In order to improve the wavenumber resolution, zero-padding is used to obtain a uniform wavenumber resolution of $1 \mathrm{~m}^{-1}$ along $k_{x}$ and $k_{y}$.

In order to assess the accuracy of the reciprocity approach for evaluating the panel sensitivity functions, experimental results obtained with this approach are compared with numerical results obtained by considering the direct interpretation of these functions (as described at the end of Sec. 2.1). This comparison allows validating the uniform mesh of $37 \times 27$ points used for the spatial DFT of the panel velocity field. The numerical model used for this study is described in Ref. [12].

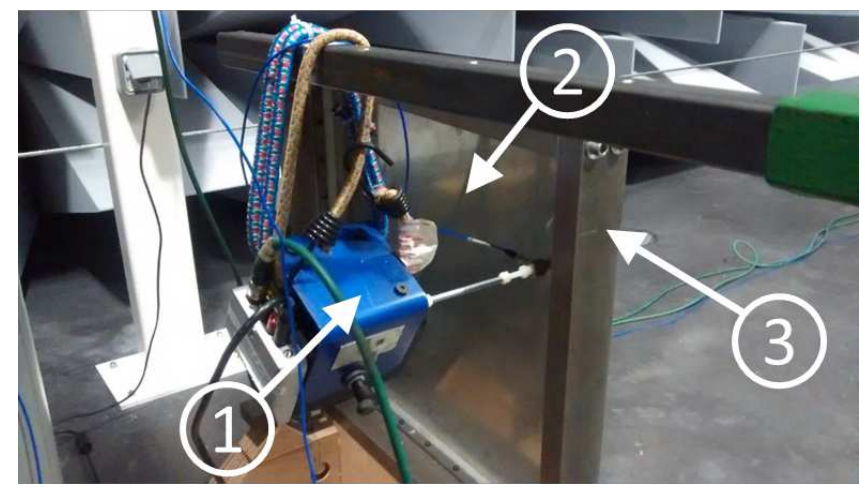

Fig. 4. Experimental setup, plate excited by a shaker to determine $H_{v} .1$ - shaker with impedance head. 2 - plate. 3 - frame.

Fig. 4 shows the experimental setup used to measure the sensitivity functions. The plate was glued on thin blades and fixed on a frame as described in [10] to reproduce simply supported boundary conditions. To determine the velocity sensitivity functions $H_{v}$, the plate was excited by a normal force at point $\mathbf{x}_{M}$ of coordinates $(x=0.18 \mathrm{~m}, y=0.09 \mathrm{~m})$. This force was applied using a TMS SmartShaker K2007E01 with integrated amplifier, which was fed with a swept sine over the considered frequency range and the force was measured using an impedance head PCB288D01. An adapter was used between the impedance head and the plate reducing the area of mechanical coupling to approximately a $5 \mathrm{~mm}$ diameter circle. The vibratory response of the panel was measured on the grid of $37 \times 27$ points with a single point laser vibrometer (PSV-300 Polytec) and a time Fourier transform was directly performed in the post-processing software with ten linear averages. 

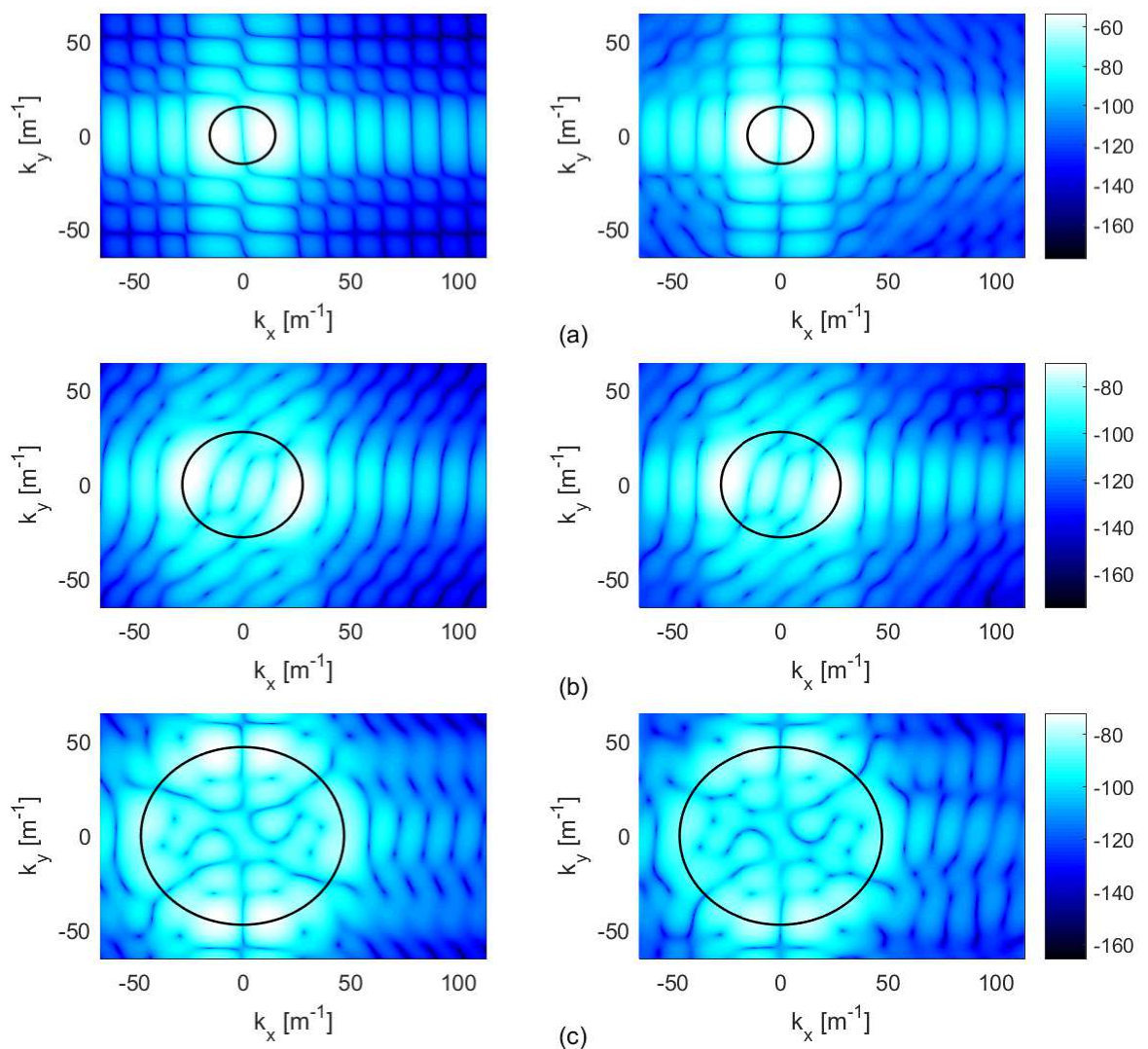

Fig. 5. Velocity sensitivity functions at point $\mathbf{x}_{M},\left|H_{v}\right|^{2}\left(\mathrm{~dB}\right.$, ref. $\left.1 \mathrm{~m}^{2} \cdot \mathrm{s}^{-2}\right)$ : direct calculation (left), experimental reciprocity approach (right). (a) $f=178 \mathrm{~Hz}$. (b) $f=$ $600 \mathrm{~Hz}$. (c) $f=1710 \mathrm{~Hz}$. - , circle of radius $k_{f}$. 
Fig. 5 shows the velocity sensitivity functions $H_{v}$ obtained with the direct numerical calculation and the reciprocal approach using experimental data. They are provided for three different frequencies, the lowest corresponding to the $(2,1)$ vibration mode frequency (Fig. 5(a)) and the two others being off-resonance cases (Figs. 5(b) and 5(c)). The circle of radius $k_{f}=\left(\omega^{2} \rho h / D\right)^{1 / 4}$, corresponding to the natural flexural wavenumber, is also indicated in Fig. 5. The numerical and experimental results are generally in good agreement. One can observe that the experimental sensitivity functions are slightly overestimated at the $(2,1)$ vibration mode frequency compared to the numerical results. This can be explained by the fact that the modal damping loss factor has been estimated from the response of the plate to a shaker excitation. The added mass from the impedance head and adapter possibly had an influence on the evaluation of the damping of the $(2,1)$ mode.

A good agreement is particularly noticed within the flexural wavenumber circle (delineated by a continuous line). Again, mainly the values in the flexural wavenumber circle contribute to the plate's vibration response to a TBL. However, the experimental sensitivity functions are also correctly estimated for wavenumbers higher than the flexural wavenumber.

\section{Comparison with measurements in an anechoic wind tunnel}

Finally, the proposed methodology is fully conducted as described in Sec. 3 to estimate the response of the plate $G_{v v}$ and compare it with direct measurements in an anechoic wind tunnel. The proposed methodology requires data in the form of CSD functions expressed in the wavenumber domain to describe the TBL excitation. The considered data describing the wall-pressure fluctuations are first presented in Sec. 5.1, then the response of the plate predicted with the proposed approach is compared to wind tunnel measurements in Sec. 5.2.

\subsection{Measurement of the wall-pressure fluctuations in an anechoic wind tunnel}

Several analytical and semi-empirical models of a TBL excitation can be found in the literature such as Corcos and Chase models $[13,14]$, but currently no model accurately predicts the wall-pressure fluctuations induced by a TBL excitation. For the sake of validating the proposed methodology by comparison with actual measurements in an anechoic wind tunnel, the CSD functions were estimated from measurements of wall-pressure fluctuations of the TBL reproduced in the wind tunnel. The wall-pressure fluctuations have been measured using the spiralshaped rotating antenna introduced by Robin et al. [15] at a flow velocity of $U_{\infty}=40 \mathrm{~m} . \mathrm{s}^{-1}$ (see Fig. 6(b)). To help the TBL develop, a sandpaper strip was glued at the end of the convergent.

The pattern over which the 61 microphones are positioned (see Fig. 6(a)) has been designed to keep the microphone density reasonable and so that each 


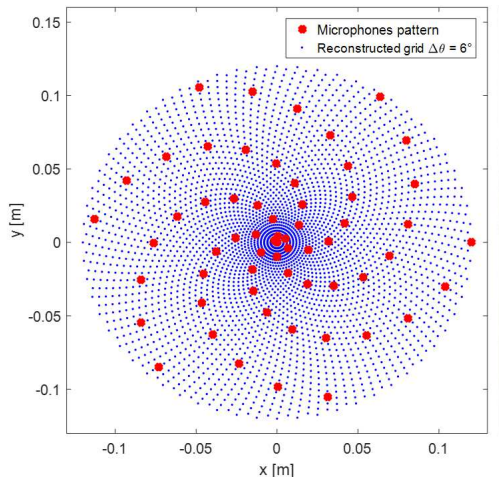

(a)

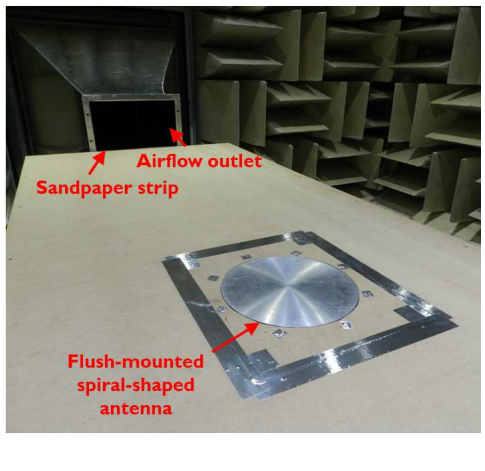

(b)

Fig. 6. Measurement of the wall-pressure fluctuations. (a) Antenna pattern and illustration of the reconstructed grid when rotating the antenna with $\Delta \theta=6^{\circ}$ increments. (b) Spiral-shaped array flush-mounted in the wind tunnel.

microphone has a different radial position $r_{m}(m=1: M$, with $M=61)$ with a radial resolution $\Delta r=2 \mathrm{~mm}$. Measurements made following $N$ consecutive rotations $\theta_{n}(n=1: N)$ allowed reaching an angular resolution $\Delta \theta=360 / N^{\circ}$ and thereby reconstructing a high density microphone array (as illustrated in Fig. 6(a)) at a post-processing step. This implies that the excitation is stationary in time and spatially homogeneous. The wavenumber-frequency CSD functions are estimated as follows [16]:

$$
\bar{G}_{p_{b} p_{b}}(\boldsymbol{k}, f)=\sum_{m=1}^{M} \sum_{n=1}^{N} \bar{G}_{p_{b} p_{b}}\left(r_{m}, \theta_{n}, f\right) e^{-j\left(k_{x} r_{m} \cos \theta_{n}+k_{y} r_{m} \sin \theta_{n}\right)} r_{m} \Delta r \Delta \theta
$$

where $\bar{G}_{p_{b} p_{b}}\left(r_{m}, \theta_{n}, f\right)$ is the one-sided spatial CSD function of the wall-pressure. The spatial CSD functions have been estimated from the measured wall-pressure in the time domain $\bar{p}_{b}\left(r_{m}, \theta_{n}, t\right)$ at each position $\left(r_{m}, \theta_{n}\right)$ using "cpsd" MATLAB command. The spiral-shaped array was flush-mounted $1.8 \mathrm{~m}$ away from the convergent and 30 seconds acquisitions were performed for each of the $N=180$ rotations. The reconstructed grid theoretically allows reaching a maximum wavenumber of $1570 \mathrm{~m}^{-1}$, which is well above the limits of the previously defined wavenumber domain $\Omega_{\boldsymbol{k}}$. Applying Eq. (14) leads to an estimation of the wavenumber-frequency CSD functions at the center of the array $(x=0, y=0)$. The obtained estimation of the wall-pressure CSD function is presented for 3 frequencies in Fig. 7. 


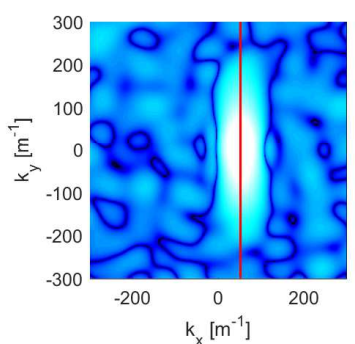

(a)

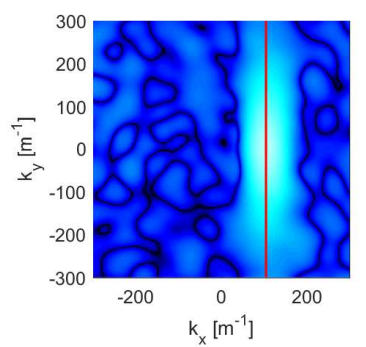

(b)

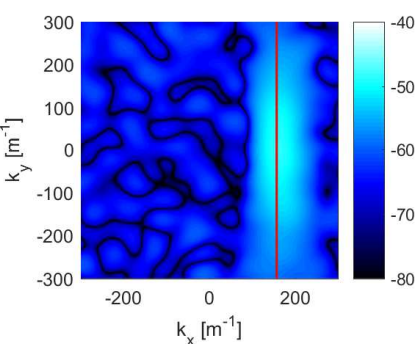

(c)

Fig. 7. Wall-pressure CSD functions $\bar{G}_{p_{b} p_{b}}(\boldsymbol{k}, f)\left(\mathrm{dB}\right.$, ref. $\left.1 \mathrm{~Pa}^{2}\right)$ estimated from measurements. (a) $f=250 \mathrm{~Hz}$. (b) $f=500 \mathrm{~Hz}$. (c) $f=750 \mathrm{~Hz}$. Vertical red line, $k_{x}=k_{c}$.

\subsection{Vibration response}

The vibration response of the plate estimated from the proposed approach is finally compared to direct measurement in the wind tunnel. On one hand, measurements in the wind tunnel were performed with a plate similar to the one used in the previous section (similar dimensions, material and boundary conditions). The plate was flush-mounted at the location of the previously mounted spiralshaped array (as shown in Fig. 8) and excited at a flow velocity of $U_{\infty}=40 \mathrm{~m} . \mathrm{s}^{-1}$ (aside from replacing the spiral-shaped array with the plate, the setup remained unchanged).

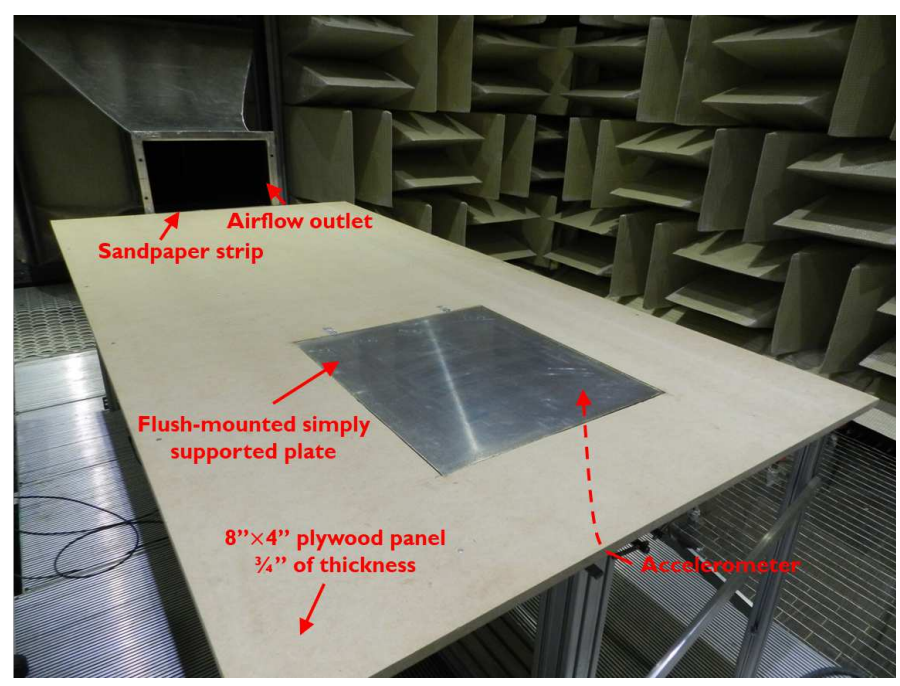

Fig. 8. Experimental setup used to measure the vibration response of the plate to the TBL reproduced in the wind tunnel. 
The vibration velocity of the plate was measured at point $\mathbf{x}_{M}$ using an accelerometer and time signals were extracted from the post-processing software. The velocity ASD function $G_{v v}$ was estimated using the "cpsd" MATLAB command.

On the other hand, the velocity ASD function was estimated by applying the proposed methodology using Eq. (6) and the measured wall-pressure CSD function $\bar{G}_{p_{b} p_{b}}(\boldsymbol{k}, f)$.

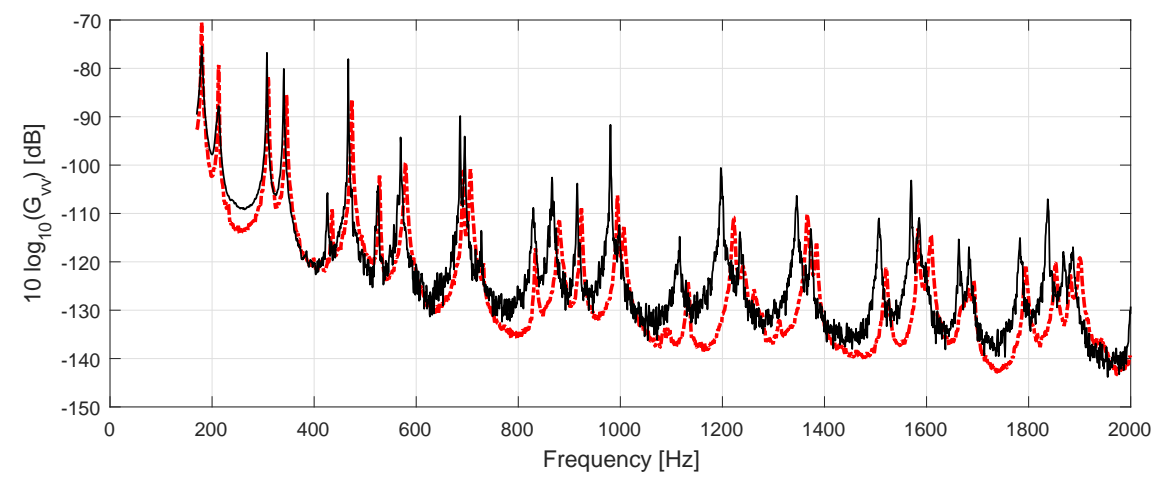

Fig. 9. Velocity ASD functions $G_{v v}\left(\mathrm{~dB}\right.$, ref. $\left.1 \mathrm{~m}^{2} \cdot \mathrm{s}^{-2} \cdot \mathrm{Hz}^{-1}\right)$ : wind tunnel measurements (dashed red line) vs. experimental reciprocity approach (continuous black line).

The plate velocity ASD function measured in the anechoic wind tunnel room at point $\mathbf{x}_{M}$ is compared to the result obtained with the proposed method in Fig. 9. The two curves are in very good agreement, which shows that for the considered test case, the velocity response of the plate can be fairly well estimated experimentally by simply applying a normal effort at the point of interest. Slight shifts of the resonance peaks in the high frequency range are noticed. They can be explained by the fact that the sensitivity functions were measured on a plate which is slightly different to the one mounted in the wind tunnel to directly measure the response. Differences is terms of peak values of the velocity ASD functions can also be noticed. Since the proposed method is purely experimental, it includes the structural damping of both plates. These differences in peak values are therefore again linked to the experimental set-ups.

\section{Conclusion}

In this paper, a methodology for characterizing the response of flat panels to a turbulent boundary layer excitation without using common measuring techniques (i.e., wind tunnel, in situ measurements) was proposed. This approach is 
based on the mathematical formulation of the random excitation problem in the wavenumber domain. This formulation indicates that the panel response at point $\mathbf{x}$ on the panel to a random field depends on two quantities in the wavenumber domain: the wall-pressure cross spectral density function of the excitation and so-called 'sensitivity functions' at point $\mathbf{x}$ which characterize the panel. Using the reciprocity principle, it has been shown that these functions can be determined from the panel velocity field in the wavenumber domain when it is excited by a normal force of unit amplitude at the point of interest $\mathbf{x}$. The sensitivity functions can be estimated easily by experiment based on this reciprocal interpretation.

The proposed approach avoids using very costly facilities or measurements techniques such as wind tunnels or in situ measurements and is fairly simple to apply. It should however be underlined that the main limitations of the proposed approach rely on the assumptions of the mathematical formulation of the problem: the system should be linear (i.e., elastic material, small deformations) and time invariant. Also, a good estimation of the considered excitation is required. Models provided in the literature can be used but may lead to unsatisfactory results when it comes to comparing the proposed approach with other measuring techniques involving an actual reproduction of a turbulent boundary layer. This is the main reason why the wall-pressure fluctuations have been measured in this paper, but it is not mandatory to apply the method.

From a practical point of view, an accurate experimental reproduction of the reciprocal source is required (i.e., normal force). A vibration measuring device is also needed to determine the vibratory response over the entire panel. A scanning laser vibrometer was used in this study, but with the recently developed full-field vibration measuring techniques (such as digital image correlation or deflectometry [17]), time of experiment could be significantly reduced in the future.

The method has been validated numerically and experimentally for the considered test case. Comparisons of numerical and experimental results have shown that the sensitivity functions are well estimated both inside and outside the natural flexural circle in the wavenumber domain. The proposed approach has finally been confronted to direct measurements in an anechoic wind tunnel. Results have shown that a fairly good estimate of the vibration response can be obtained by applying the proposed methodology. This methodology has been extended to the prediction of acoustic quantities [12] such as the radiated power. In the near future, it will be applied to determine the acoustic response of a panel to a turbulent boundary layer.

\section{References}

1. M. Aucejo, L. Maxit, J.-L. Guyader, "Experimental simulation of turbulent boundary layer induced vibrations by using a synthetic array", J. Sound Vib. 331(16), 3824-3843 (2012). doi:10.1016/j.jsv.2012.04.010 
2. A. Berry, R. Dia, O. Robin, "A wave field synthesis approach to reproduction of spatially correlated sound fields", J. Acoust. Soc. Am. 131(2), 1226-1239 (2012). doi:10.1121/1.3675942

3. O. Robin, A. Berry, S. Moreau, "Reproduction of random pressure fields based on planar nearfield acoustic holography", J. Acoust. Soc. Am. 133(6), 3885-3899 (2013). doi:10.1121/1.4802898

4. T. Bravo, C. Maury, "The experimental synthesis of random pressure fields: Methodology", J. Acoust. Soc. Am. 120(5), 2702-2711 (2006). doi:10.1121/1. 2354008

5. T. Bravo, C. Maury, "A synthesis approach for reproducing the response of aircraft panels to a turbulent boundary layer excitation", J. Acoust. Soc. Am. 129(1), 143153 (2011). doi:10.1121/1.3514530

6. C. Maury, T. Bravo, "Focussed Synthesis of a Turbulent Boundary Layer Excitation", 22nd AIAA/CEAS Aeroacoustics Conference, Aeroacoustics Conferences, pp. 1-12 (2013). doi:10.2514/6.2016-2763

7. C. Maury, P. Gardonio, S. J. Elliott, "A wavenumber approach to modelling the response of a randomly excited panel, part 1: general theory", J. Sound Vib. 252(1), 83-113 (2002). doi:10.1006/jsvi.2001.4028

8. Y. K. Lin, "Probabilistic theory of structural dynamics", p. 207, McGraw-Hill, New York (1967).

9. F. J. Fahy, "Some Applications of the Reciprocity Principle in Experimental Vibroacoustics", Acoustical Physics 49(2), 217-229 (2003). doi:10.1134/1.1560385

10. O. Robin, J-D. Chazot, R. Boulandet, M. Michau, A. Berry, N. Atalla, "A plane and thin panel with representative simply supported boundary conditions for laboratory vibroacoustic test", Acta Acust. United Ac. 102(1), 170-182 (2016). doi:10.3813/ AAA.918934

11. G. M. Corcos, "Resolution of Pressure in Turbulence", J. Acoust. Soc. Am. 35(2), 192-199 (1963). doi:10.1121/1.1918431

12. C. Marchetto, L. Maxit, A. Berry, O. Robin, "Vibroacoustic response of panels under diffuse acoustic field excitation from sensitivity functions and reciprocity principles", J. Acoust. Soc. Am. 141(6), 4508-4521 (2017). doi:10.1121/1.4985126

13. T. S. Miller, J. M. Gallman, and M. J. Moeller, "Review of Turbulent Boundary Layer Models for Acoustic Analysis", 49th AIAA Aerospace Sciences Meeting, pp. 1-20 (2011). doi:10.2514/1.C031405

14. A. Caiazzo, R. D'Amico, W. Desmet, "A Generalized Corcos model for modelling turbulent boundary layer wall pressure fluctuations", J. Sound Vib. 372, 192-210 (2016). doi:10.1016/j.jsv.2016.02.036

15. O. Robin, S. Moreau, T. Padois, A. Berry, "Measurement of the wavenumberfrequency spectrum of wall pressure fluctuations: spiral-shaped rotative arrays with pinhole-mounted quarter inch microphones", 19th AIAA/CEAS Aeroacoustics Conference, Aeroacoustics Conferences, pp. 1-18 (2013). doi:10.2514/6.2013-2058

16. B. Arguillat, D. Ricot, C. Bailly, G. Robert, "Measured wavenumber: Frequency spectrum associated with acoustic and aerodynamic wall pressure fluctuations", J. Acoust. Soc. Am. 128(4), 1647-1655 (2010). doi:10.1121/1.3478780

17. M. Grédiac, F. Hild, "Full-Field Measurements and Identification in Solid Mechanics", Chap. 3 and 6, ISTE Ltd. and John Wiley \& Sons Inc., London (2013). 